\title{
Micro-Brillouin Spectroscopy Applied to the Glass Transition of Anti-inflammatory Egonol
}

\author{
Tae Hyun Kim and Jae-Hyeon Ko* \\ Department of Physics, Hallym University, Chuncheon 200-702, Korea \\ Eun Mi Kwon and Jong-Gab Jun \\ Department of Chemistry and Institute of Natural Medicine, Hallym University, Chuncheon 200-702, Korea
}

(Received July 22, 2010 : revised September 6, 2010 : accepted September 9, 2010)

\begin{abstract}
The acoustic properties of anti-inflammatory egonol were investigated by using micro-Brillouin scattering spectroscopy, by use of a 6-pass tandem Fabry-Perot interferometer and an optical microscope specially modified for spectroscopic purposes. The measured Brillouin spectrum was composed of a central peak centered at zero and a Brillouin doublet arising from the longitudinal acoustic waves, i.e. propagating density fluctuations. For the first time, the glass transition of egonol was identified to be about $5^{\circ} \mathrm{C}$ at which the Brillouin peak position and the half width showed abrupt changes. The substantial damping of acoustic phonons of egonol near the glass transition temperature indicated that the contribution of internal relaxation processes such as small-amplitude librations of side chains to the damping of acoustic phonons may be substantial depending on the internal structure of molecules.
\end{abstract}

Keywords: Fabry-Perot interferometer, Brillouin scattering, Acoustic waves, Egonol, Glass transition OCIS codes : (120.2230) Fabry-Perot; (120.6200) Spectrometers and spectroscopic instrumentation; (290.5830) Scattering, Brillouin; (300.6330) Spectroscopy, inelastic scattering including Raman

\section{INTRODUCTION}

Brillouin spectroscopy has been a conventional experimental method for studying low-energy excitations such as long-wavelength acoustic phonons, spin density waves, and soft modes in condensed matter. Since the energy scales of these excitations are located in the frequency range of $\mathrm{GHz} \sim \mathrm{THz}$, conventional grating-based spectrometers cannot be used to probe this low-frequency range. The Fabry-Perot etalon is the most suitable optical component for this purpose and comprises an essential part in the Brillouin spectrometer $[1,2]$. The etalon, consisting of two parallel mirrors with high reflectivity, transmits light when the mirror spacing is a multiple of half of its wavelength. Scanning the mirror spacing with, for example, piezoelectric devices enables one to measure a certain frequency range of a light spectrum. The one-pass Fabry-Perot interferometer (FPI), along with the advent of coherent laser light sources, played an important role in the early history of
Brillouin spectroscopy, but the measured spectra were characterized by a low contrast and a narrow free spectral range (FSR). The development of a multi-pass tandem FabryPerot interferometer (T-FPI) made it possible to get a contrast higher than $10^{9}$ and a wide FSR up to $1 \mathrm{THz}$ [3].

However, there have been two deficiencies in applying T-FPI to condensed matter. First, it takes a relatively long acquisition time to get a spectrum due to the limited usage of the scattered light in T-FPI. This makes it almost impossible to monitor acoustic properties in a real-time manner. Second, it is difficult to investigate small samples of which the size is the order of $\mu \mathrm{m}$. The first problem can be overcome by using the so-called angular dispersiontype Fabry-Perot interferometer combined by an area detector $[4,5]$. The second difficulty can be solved by adopting an optical microscope in the T-FPI [6]. By using this micro-Brillouin spectroscopy, it is possible to probe microheterogeneity of condensed matter [7], surface elastic waves on inhomogeneous opaque materials [6], or eigenvi-

\footnotetext{
*Corresponding author: hwangko@hallym.ac.kr

Color versions of one or more of the figures in this paper are available online.
} 
brations of nanospheres [8]. Recently, compact temperature chambers developed for optical microscopes are commercially available. They can be used to cover a wide temperature range from liquid He-temperature to higher than 150 $0^{\circ} \mathrm{C}$. The combination of the micro-Brillouin spectroscopy and these temperature stages opens a new possibility for investigating elastic properties of various types of condensed matter with various thermal histories.

The present study is devoted to reporting the setup of the micro-Brillouin spectrometer and its application to the glass-transition process of anti-inflammatory egonol (5-(3hydroxypropyl)-7-methoxy-2-(3,4-methylenedioxyphenyl)be nzofuran) molecules. The study of glass transitions of pharmaceutical materials such as egonol has attracted great attention from the viewpoint of fundamental physics as well as applications[9]. The glass transition is one of the fundamental phenomena that are not understood clearly due to various structural and dynamic complexities [10-11]. The detailed studies of various materials including pharmaceuticals may give us more insight into universal features of the glass transition. In addition, glassy pharmaceutical materials have recently been studied intensively because a vitreous state is regarded as more effective than the crystalline state due to the higher dissolving speed and thus higher bioavailability [9]. Egonol has been known to show variety of biological properties including insecticidal, fungicidal, antimicrobial, antisweet, antiproliferative, cytotoxic and antioxidant properties. However, systematic investigation of its physical properties, including its elastic properties, is quite incomplete. The present study reports on the change in the elastic properties during the glass transition process of egonol and determination of the glass transition temperature for the first time by using high-resolution microBrillouin scattering spectroscopy.

\section{EXPERIMENT}

Figure 1 shows the schematic diagram of the microBrillouin spectrometer consisting of a 6-pass tandem FabryPerot interferometer (JRS Sci. Instrum.) and an optical microscope (BX41, Olympus). The T-FPI consists of two FPIs (FPI1 and FPI2) which are inclined to each other by a certain angle, resulting in slightly different FSRs. Complete dynamic synchronization between the two FPI's is maintained over a large scanning range. This condition enables one to suppress neighboring transmission peaks other than the synchronized one, and thus to effectively increase the FSR up to $1 \mathrm{THz}$ [3]. The interferometer is scanned by means of a piezoelectric transducer. In addition, the distance between the two mirrors is sensed using a capacitive displacement transducer. In order to check the correctness of the frequency scale of the transmission spectrum, one of standard samples of which the correct sound velocity is reported is selected, depending on FSR. The measured Brillouin spectrum is used to calculate the sound velocity, which is compared to the exact value. In the present case, the Brillouin spectrum of high-purity ethanol (purity of $99.9 \%$ ) was measured at $24^{\circ} \mathrm{C}$, and the calculated sound velocity was found to be consistent with the literature value. Since the incident light passes through these two FPIs 6 times before it reaches the photomultiplier tube as is shown in Fig. 1(a), the contrast can become extremely high, much higher than $10^{9}$.

A commercially available microscope was specially modified to carry out the micro-Brillouin scattering experiment. A metal hollow cylinder of 70-mm diameter and 40-mm thick was inserted between the objective lens and the binocular part of the microscope. This cylinder has three side holes with a diameter of $5 \mathrm{~mm}$, through which a small coated mirror of $3.00 \times 4.24 \mathrm{~mm}^{2}$ was inserted in the hollow space of $25-\mathrm{mm}$ diameter. Fig. 1(b) displays a horizontal cross-sectional view of the hollow metal disk inserted in the microscope for micro-Brillouin spectroscopy. A laser beam coming from a diode-pumped solid state laser (Monopower-532-100-SM, Alphalas) at a wavelength of $\lambda=532 \mathrm{~nm}$ enters one of the three holes, is reflected from the inserted mirror downward to the sample via an objective lens. The scattered light from the sample is collected by the same objective lens and then is headed to T-FPI. A compact cryostat (THMS600, Linkam) was installed on the stage of the microscope to

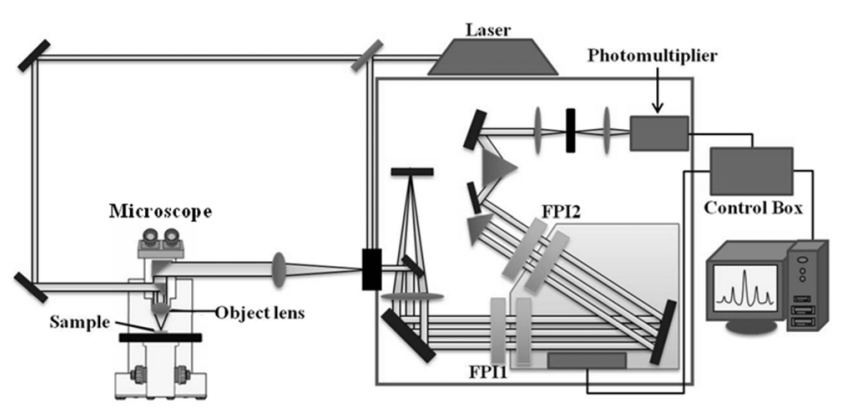

(a)

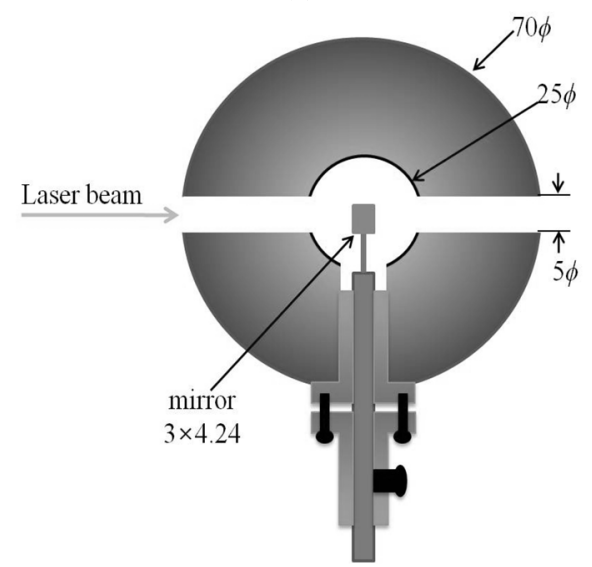

(b)

FIG. 1. (a) A schematic diagram of the micro-Brillouin spectrometer used in the present study. (b) A horizontal cross-sectional view of the hollow metal disk inserted in the microscope for micro-Brillouin spectroscopy. 


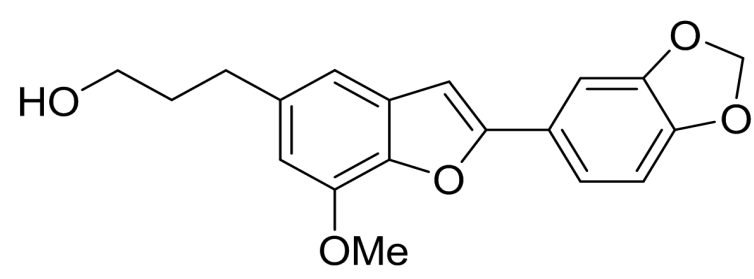

FIG. 2. The chemical structure of egonol molecules.

cover a wide temperature range between -200 and $600^{\circ} \mathrm{C}$. The spot diameter $d$ of the laser beam at the focal point of the objective lens is determined by $(4 / \pi) \lambda f / D_{L}$, where $\lambda, f$, and $D_{L}$ are the wavelength, the focal length of the objective lens, and the diameter of the incident laser beam, respectively. If we input into this equation $\lambda=532 \mathrm{~nm}, f$ $=9 \mathrm{~mm}$, and $D_{L} \sim 1 \mathrm{~mm}$, the estimated diameter of the focused beam on the sample is $\sim 6 \mu \mathrm{m}$. This means that one can discriminate elastic microheterogeneity at a spatial resolution of $\sim 6 \mu \mathrm{m}$.

The molecular structure of egonol is shown in Fig. 2. Benzo $[b]$ furan natural products isolated from the Styracaceae family show variety of biological activities including insecticidal, fungicidal, antimicrobial, antisweet, antiproliferative, cytotoxic and antioxidant properties [12]. Egonol, 5-(3-hydroxypropyl)-7-methoxy-2-(3,4-methylenedioxyphenyl)benzofuran, was first isolated in 1915 from the seed oil of Styrax japonicum [13] and first synthesized by Kawai [14] condensing an o-hydroxybenzaldehyde with an $\alpha$-chlorophenylacetic acid and is known to be an effective pyrethrum synergist [15]. In the present study, egonol was synthesized in 5 steps with $74 \%$ overall yield from vanillin by using Sonogashira coupling reaction according to Ref.[16]. For the measurement of the glass transition of egonol, the synthesized egonol powder was melted completely at $120^{\circ} \mathrm{C}$ above the melting point $\left(\sim 110^{\circ} \mathrm{C}\right)$ and then quenched to liquid nitrogen temperature $\left(-196^{\circ} \mathrm{C}\right)$ at a cooling rate higher than $20^{\circ} \mathrm{C} / \mathrm{min}$. The Brillouin spectrum was then measured during the heating process. Egonol has several internal degrees of freedom such as several side chains, hydroxyl group, etc., as can be seen from Fig. 2. Related internal relaxation (overdamped vibrations) processes are expected to contribute to the acoustic behaviors in addition to the structural relaxations [17].

\section{RESULTS AND DISCUSSION}

Figure 3 shows the spectrum of the laser beam measured at $\mathrm{FSR}=25 \mathrm{GHz}$ by inserting weak laser light directly into T-FPI and recording the spectrum. Since the laser linewidth is only $10 \mathrm{MHz}$, the spectrum of the incident laser beam can be regarded as a delta function in T-FPI, and the recorded spectrum can safely be considered as the instrumental transmission function of the interferometer. The measured spectrum is theoretically 3-times-3-pass Airy

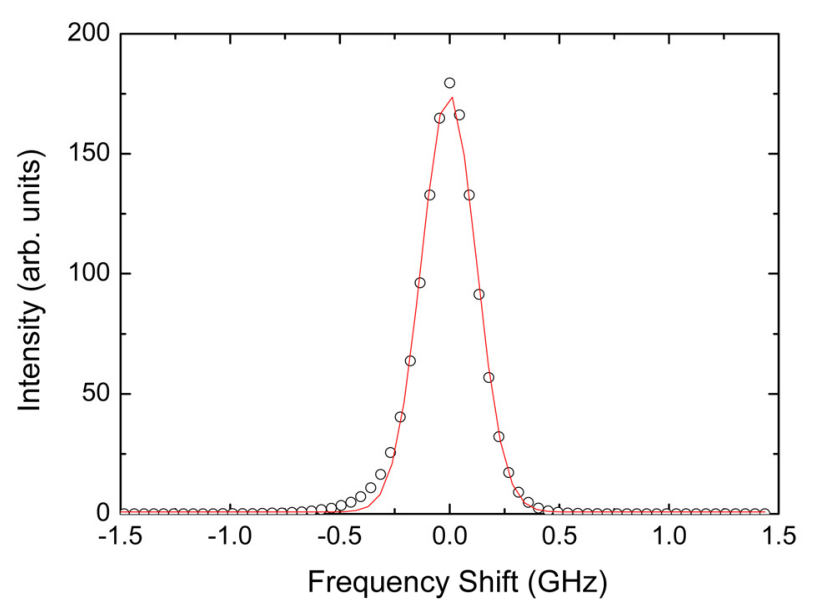

FIG. 3. The measured instrumental transmission function (white circles) and the fitted result (red solid line) by a Gaussian function. See the text for details.

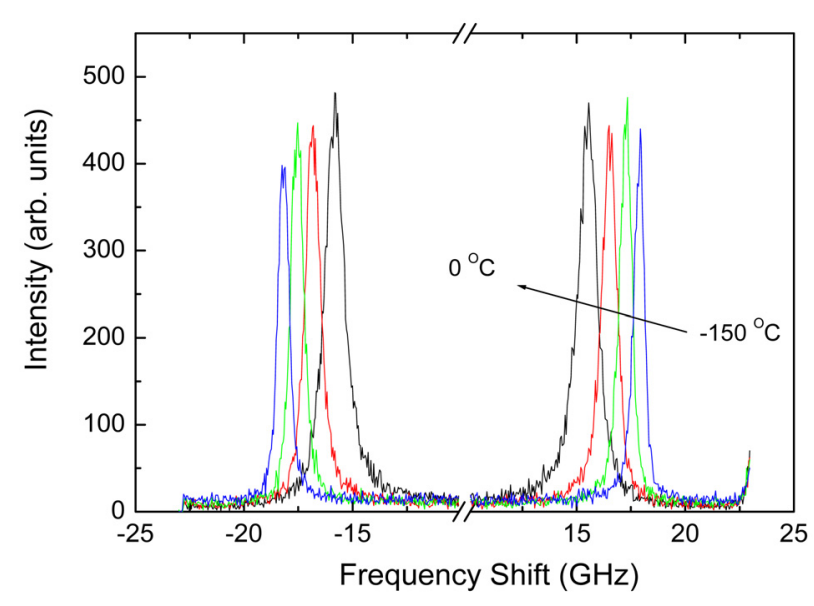

FIG. 4. Measured Brillouin spectra of egonol. The measurement temperatures are $-150,-100,-50,0^{\circ} \mathrm{C}$.

function which can be well approximated by a Gaussian function. The solid line of Fig. 3 denotes the best-fitted result by a Gaussian function, from which the full-width at half-maximum (FWHM) was obtained to be $0.29 \mathrm{GHz}$. This means the finesse of T-FPI at this FSR is about 85 .

Figure 4 displays the measured Brillouin spectrum of egonol as a function of temperature. The Brillouin spectrum consists of one Brillouin doublet corresponding to the propagating longitudinal acoustic (LA) waves and a weak central peak centered at zero frequency. The peak position shifts to lower-frequency and the linewidth increases upon heating. The scattered spectrum due to LA waves can be approximated by that of a damped harmonic oscillator as follows.

$$
I(v)=\frac{I_{0}}{\pi} \frac{v_{B}^{2} \Gamma_{B}}{\left(v^{2}-v_{B}^{2}\right)^{2}+v^{2} \Gamma_{B}^{2}} .
$$


In this expression, $v$ is the frequency, $I_{0}$ the proportional constant, and $v_{\mathrm{B}}$ and $\Gamma_{\mathrm{B}}$ are the frequency position and FWHM of Brillouin peaks. If $v_{\mathrm{B}}$ is much larger than $\Gamma_{\mathrm{B}}$ as is usual, the above form is well approximated by a Lorentzian function given by

$$
I(v)=\frac{I_{0}}{\pi} \frac{\Gamma_{B} / 4}{\left(v-v_{B}\right)^{2}+\Gamma_{B}^{2} / 4} .
$$

This Lorentzian function should be convoluted to the instrumental function in order to fit the experimental data. The experimental data were finally fitted by using the following Voigt function, which is a convolution of the Lorentzian and the Gaussian functions.

$$
I(v)=A \frac{\Gamma_{B}}{\Gamma_{G}^{2}} \int_{-\infty}^{\infty} \frac{e^{-t^{2}}}{\left(\sqrt{\ln (2)} \frac{\Gamma_{B}}{\Gamma_{G}}\right)^{2}+\left(\sqrt{4 \ln (2)} \frac{v-v_{B}}{\Gamma_{G}}-t\right)^{2}} d t
$$

In this equation, $A$ is a proportionality constant and $\Gamma_{\mathrm{G}}$ should be fixed to the FWHM of the measured instrumental function. Figure 5 exhibits one example of the fitting result for the Brillouin spectrum measured at $0^{\circ} \mathrm{C}$. Three Voigt functions, one for the central peak and two for the two Brillouin peaks, were used to fit the data, and the best-fitted result is shown as a solid line in the same figure. The three dotted lines correspond to the three fitting components, i.e. three Voigt functions.

From the fitting procedure, the Brillouin peak position $\nu_{\mathrm{B}}$ and the half width $\Gamma_{\mathrm{B}}$ could be obtained as a function of temperature. $v_{\mathrm{B}}$ is proportional to the sound velocity of the acoustic phonons while $\Gamma_{\mathrm{B}}$ is related to the acoustic damping or attenuation coefficient. Figure 6 shows the temper-

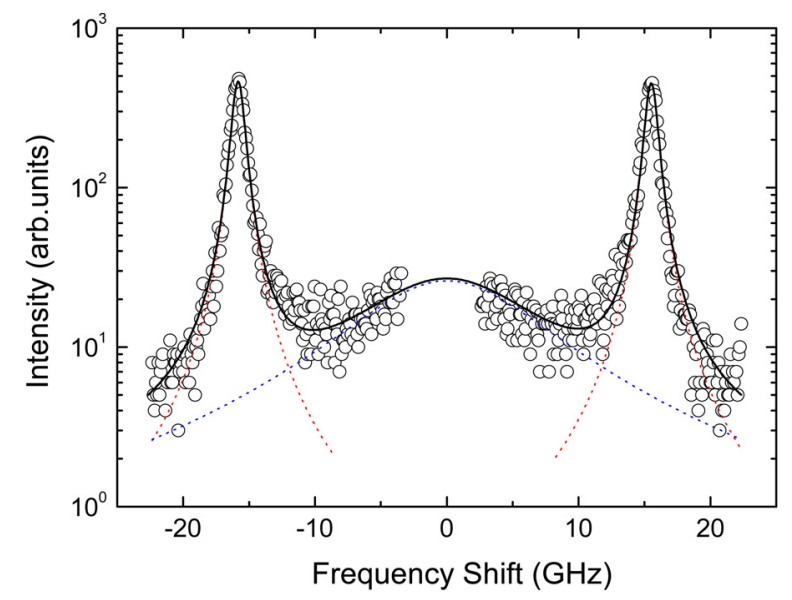

FIG. 5. The Brillouin spectrum of egonol measured at $0^{\circ} \mathrm{C}$ and the best-fitted result (solid line) by using a superposition of three Voigt functions. The dotted lines denote the three components (the LA mode and the central peak) comprising the fitting result. ature dependences of $v_{\mathrm{B}}$ and $\Gamma_{\mathrm{B}}$ of egonol. For comparison, $v_{\mathrm{B}}$ and $\Gamma_{\mathrm{B}}$ of one of the most representative pharmaceuticals, aspirin (Acetylsalicylic acid, $\mathrm{HCOO}^{-} \mathrm{C}_{6} \mathrm{H}_{4}-\mathrm{COOCH}_{3}$ ), were shown in the insets of Fig.6, which was taken from Ref. [18]. $v_{B}$ shows softening in the measured temperature range upon heating. The mild softening of $v_{\mathrm{B}}$ persists up to about $5^{\circ} \mathrm{C}$ and then the slope displays a noticeable change. This temperature is usually defined as the glass transition temperature $\left(T_{\mathrm{g}}\right)$ because at $T_{\mathrm{g}}$ the sudden change in the molecular mobility affects the viscosity and the elastic properties. In the supercooled liquid phase above $T_{\mathrm{g}}$, the change in $v_{\mathrm{B}}$ becomes steeper. The sudden change in the slope of $v_{\mathrm{B}}$ reflects in good approximation the corresponding change in the slope of the temperature dependence of the density [19]. The acoustic damping, represented by FWHM shown in Fig.6(b), begins to show a remarkable increase above $T_{\mathrm{g}}$ indicating the substantial contribution of the structural relaxation to the damping of phonons. The Brillouin data above $40^{\circ} \mathrm{C}$ could not be obtained because $v_{\mathrm{B}}$ exhibited a sudden, discontinuous increase at $\sim 40^{\circ} \mathrm{C}$

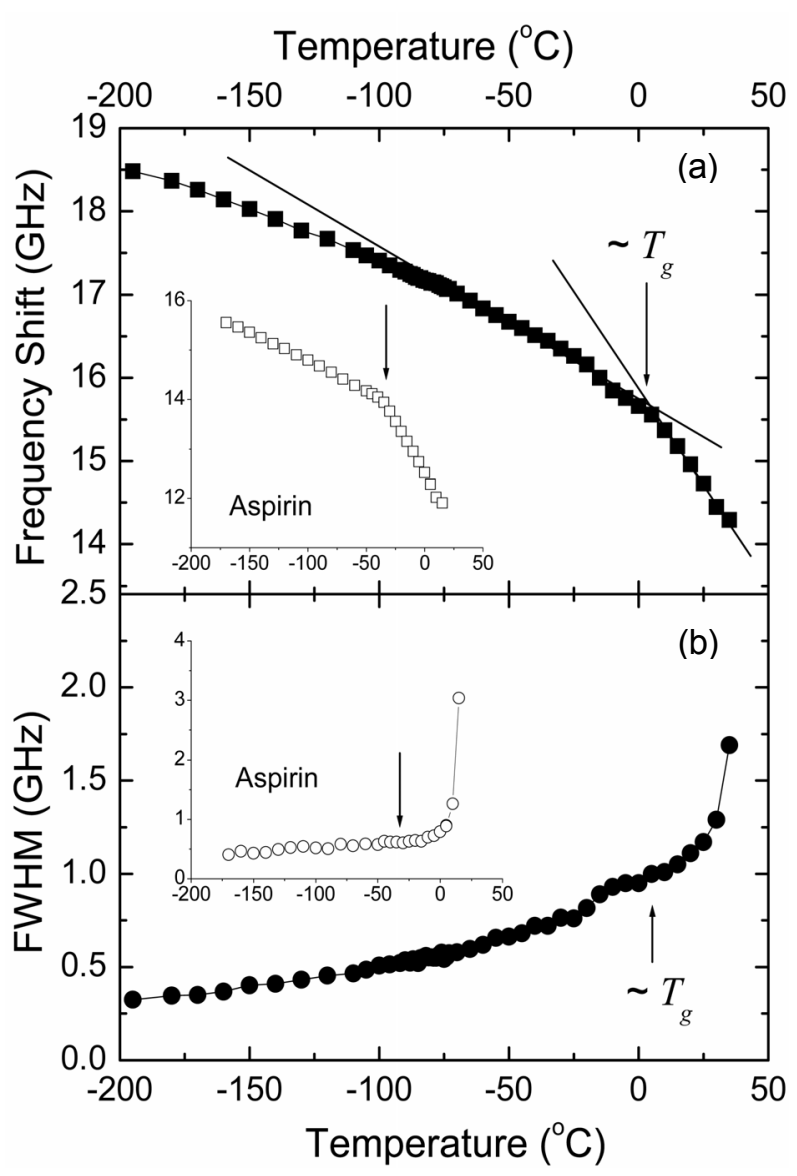

FIG. 6. The temperature dependence of (a) the Brillouin frequency shift and (b) the full-width at half-maximum (FWHM) of egonol. The lines are guide to the eyes. The glass transition temperature $\left(T_{\mathrm{g}}\right)$ is indicated by downward arrows. The insets show the same data of aspirin which were taken from Ref.[18]. 
and the supercooled egonol gradually became opaque, indicating the onset of cold-crystallization near this temperature.

Vitreous pharmaceuticals have attracted high attention due to their higher bioavailability. Moreover, vitreous drugs do not have the problem arising from different effects of crystalline pharmaceuticals due to polymorphism. In this context, the stability of the vitreous state of pharmaceuticals is very important from the viewpoint of application. $T_{\mathrm{g}}$ of egonol determined by Brillouin scattering is located below the room temperature, and the vitreous state can easily be transformed into the crystalline state. This means that the vitreous egonol is very unstable at normal conditions and that it is necessary to investigate other egonol derivatives having similar medicinal effects systematically but higher $T_{\mathrm{g}}$. One strategy may be increasing sterical constraints of egonol molecules by modifying side chains, which is an effective way to increase $T_{\mathrm{g}}$.

Finally, the acoustic properties of egonol will be briefly compared to those of aspirin and the differences will be discussed. For these two pharmaceuticals, both $v_{\mathrm{B}}$ and $\Gamma_{\mathrm{B}}$ exhibit similar temperature dependences, i.e. the slope of $v_{\mathrm{B}}$ changes substantially at $T_{\mathrm{g}}$ and $\Gamma_{\mathrm{B}}$ increases remarkably above $T_{\mathrm{g}}$. This indicates the structural relaxation process plays an important role in the acoustic dispersion as the relaxation time $\tau$ becomes closer to the inverse of the acoustic frequency in the supercooled liquid phase. At low temperatures, the relaxation time of the structural relaxation process is much longer than the inverse of the acoustic frequency, which means that the relaxation process cannot follow the acoustic waves. At high temperatures in the liquid phase, the relaxation time is very short, and we can probe the fully-relaxed acoustic properties [20]. The maximum of the acoustic damping may occur when $2 \pi v_{\mathrm{B}} \tau=1$, which could not be observed from egonol due to cold-crystallization. If we compare $\Gamma_{\mathrm{B}}$ values of two materials at $T_{\mathrm{g}}$, we can easily notice that $\Gamma_{B}$ of egonol is larger than that of aspirin by more than $60 \%$. This may be attributed to the different internal degrees of freedom. The relaxation times of internal relaxations are usually very fast and close to the inverse of the acoustic frequency at all temperatures. This suggests that internal relaxation processes will contribute substantially to the damping of phonons even in the glass phase [17]. The larger $\Gamma_{\mathrm{B}}$ observed from glassy egonol indicates that the contribution of the internal degrees of freedom to the damping of phonons is more substantial in egonol compared to aspirin because of much richer internal degrees of freedom and of the relevant low-amplitude librational motions that are remnant even in the glass phase and thus can be coupled to acoustic waves.

\section{CONCLUSION}

The acoustic properties during the glass transition process of anti-inflammatory egonol material were investigated by using micro-Brillouin scattering spectroscopy with a 6-pass tandem Fabry-Perot interferometer and a optical microscope specially modified for spectroscopic purposes. The vitreous state of egonol was prepared by quenching the liquid egonol to liquid nitrogen temperature, and the Brilloun spectrum was recorded in both glass and supercooled liquid phases upon heating. The Brillouin frequency shift (proportional to the sound velocity) showed a sudden change in the slope while the half width (related with the acoustic damping) began to increase remarkably at around $5^{\circ} \mathrm{C}$, which was defined as the glass transition temperature of egonol. The half width of egonol at and below the glass transition temperature was larger than that of aspirin, indicating that the contribution of internal relaxation processes to the damping of acoustic phonons is sensitive to the molecular structure and more substantial in egonol than in aspirin.

\section{ACKNOWLEDGMENT}

This research was supported by Basic Science Research Program through the National Research Foundation of Korea(NRF) funded by the Ministry of Education, Science and Technology (2009-0066894) and Priority Research Centers Program through the National Research Foundation of Korea (NRF) funded by the Ministry of Education, Science and Technology (2009-0094072).

\section{REFERENCES}

1. J. M. Vaughan, The Fabry-Perot Interferometer (Adam Hilger, Bristol, England, 1989).

2. G. Hernandez, Fabry-Perot Interferometers (Cambridge University Press, New York, USA, 1986).

3. J. R. Sandercock, Light Scattering in Solids III, M. Cardona and G. Güntherodt, ed. (Springer, Berlin, Germany, 1982), pp. 173.

4. J.-H. Ko and S. Kojima, "Angular dispersion-type nonscanning Fabry-Perot interferometer applied to ethanol-water mixture," J. Opt. Soc. Korea 13, 261-266 (2009).

5. J.-H. Ko and S. Kojima, "Comparison of acoustic behaviors between ethanol and partially-deuterated ethanol," J. Korean Phys. Soc. 56, 409-412 (2010).

6. Y. Takagi and K. Kurihara, "Application of a microscope to Brillouin scattering spectroscopy," Rev. Sci. Instrum. 63, 5552-5555 (1992).

7. D. H. Kim, J.-H. Ko, D. C. Feng, and S. Kojima, "Microheterogeneity and field cooling effects on PZN-4.5\%PT single crystals probed by micro-Brillouin scattering," Appl. Phys. Lett. 87, 072908 (2005).

8. Y. Li, H. S. Lim, S. C. Ng, Z. K. Wang, M. H. Kuok, E. Vekris, V. Kitaev, F. C. Peiris, and G. A. Ozin, "MicroBrillouin scattering from a single isolated nanosphere," Appl. Phys. Lett. 88, 023112 (2006).

9. G. P. Johari and D. Pyke, "On the glassy and supercooled liquid states of a common medicament: aspirin," Phys. Chem. Chem. Phys. 2, 5479-5484 (2000).

10. S. P. Das, "Mode-couplling theory and the glass transition 
in supercooled liquids." Rev. Mod. Phys. 76, 785-851 (2004).

11. J. C. Dyre, "The glass transition and elastic models of glass-forming liquids," Rev. Mod. Phys. 78, 953-972 (2006).

12. R. S. Ward, "Lignans, neolignans and related compounds," Nat. Prod. Rep. 16, 75-96 (1999).

13. M.-R. Kim, H. T. Moon, D. G. Lee, and E.-R. Woo, "A new lignin glycoside from the stem bark of Styrax japonica S. et Z.," Arch. Pharm. Res. 30, 425-430 (2007).

14. S. Kawai, T. Nakamura, and N. Sugiyama, "Synthesis of egonol," Ber. dt. Chem. Ges. 72, 1146-1149 (1939).

15. M. Takanashi and Y. Takizawa, "New benzofurans related to egonol from immature seeds of Styrax obassia," Phytochemistry 27, 1224-1226 (1988).

16. D. H. Choi, J. W. Hwang, H. S. Lee, D. M. Yang, and J.-G. Jun, "Highly effective total synthesis of benzofuran natural product egonol," Bull. Korean Chem. Soc. 29, 1594-1596 (2008).

17. A. Patkowski, J. Gapinski, G. Meier, and H. Kriegs, "Isotropic Brillouin spectra of liquids having an internal degree of freedom," J. Chem. Phys. 126, 014508 (2007).

18. J.-H. Ko, K.-S. Lee, Y. Ike, and S. Kojima, "Elastic properties of aspirin in its crystalline and glassy phases studied by micro-Brillouin scattering," Chem. Phys. Lett. 465, 36-39 (2008).

19. B. Ruta, G. Monaco, F. Scarponi, and D. Fioretto, "Brillouin light scattering study of glassy sorbitol," Phil. Mag. 88, 3939-3946 (2008).

20. J.-H. Ko and S. Kojima, "Brillouin scattering study on glass-forming ethanol," J. Non-Crystal. Solids 307-310, 154-160 (2002). 